\title{
CMB Power Spectrum Results from the South Pole Telescope
}

\author{
Christian Reichardt', ${ }^{a}$ Ryan Keisler, ${ }^{b}$ and SPT collaboration \\ ${ }^{a}$ UC Berkeley, Berkeley, CA, USA 94720 \\ ${ }^{b}$ University of Chicago, 5640 South Ellis Avenue, Chicago, IL, USA 60637 \\ E-mail:cr@bolo.berkeley.edu, rkeisler@chicago.edu
}

The South Pole Telescope (SPT) is a 10-meter telescope designed to survey the millimeter-wave sky. The telescope and its 960-element bolometric camera were successfully installed at the South Pole in 2007. Since then, the SPT has imaged 2200 square degrees of the sky with low noise and arcminute resolution. We report on the CMB power spectrum results from SPT. In conjunction with data from the WMAP satellite, the new SPT data leads to a 6 sigma detection of gravitational lensing in the CMB. The SPT+WMAP data also improve constraints on the shape of the primordial power spectrum with implications for inflationary models. Finally, the SPT+WMAP data yield measurements of the primordial helium abundance and the number of relativistic particle species in the early Universe.

XXIst International Europhysics Conference on High Energy Physics

21-27 July 2011

Grenoble, Rhônes Alpes France

\footnotetext{
* Speaker.
} 


\section{Introduction}

Measurements of temperature anisotropy in the cosmic microwave background (CMB) are among the most informative and robust probes of cosmology. The acoustic oscillations of the primordial plasma have been measured on degree scales $(\ell \lesssim 500)$ with cosmic-variance-limited precision by the WMAP [1], yielding a wealth of cosmological information [1]. The CMB temperature anisotropy power falls exponentially to smaller scales. The reduction in CMB power is due to the diffusion of photons in the primordial plasma and is often referred to as Silk damping [2]. This "damping tail" of the primary CMB anisotropy has been measured by a number of experiments, notably ACBAR [3], QUaD [4], and ACT [5].

Measurements of the CMB damping tail, in conjunction with WMAP's measurements of the degree-scale $\mathrm{CMB}$ anisotropy, provide a powerful probe of early-universe physics. The damping tail measurements significantly increase the angular dynamic range of CMB measurements and thereby improve the constraints on inflationary parameters such as the scalar spectral index and the amplitude of tensor fluctuations. Measurements of the angular scale of the damping can constrain the primordial helium abundance and the effective number of relativistic particle species during the radiation-dominated era. Finally, the damping tail is altered at the few-percent level by gravitational lensing of the $\mathrm{CMB}$, and is therefore sensitive to matter fluctuations at intermediate redshifts.

In this conference, we have presented a measurement of the CMB damping tail using data from the SPT [6, hereafter K11]. The data were taken at $150 \mathrm{GHz}$ during 2008 and 2009 and cover approximately 790 square degrees of sky. The K11 power spectrum is the best measurement of the CMB temperature anisotropy across a multipole range $650<\ell<3000$ (angular scales of $\left.4^{\prime}<\theta<16^{\prime}\right)$.

We describe the SPT survey in $\$ 2$. The observations, analysis and resulting bandpowers are presented in $\S 3$. We examine the cosmological implications in $\S 4$, and conclude in $\S 5$.

\section{The SPT Survey}

The SPT is a 10-meter diameter off-axis Gregorian telescope located at the South Pole (left panel of Figure 1). The current receiver is equipped with 960 horn-coupled spiderweb bolometers with superconducting transition-edge sensors. The sensors are read out using frequency multiplexing. The receiver included science-quality detectors at frequency bands centered at approximately 150 and $220 \mathrm{GHz}$ in 2008, and at 95, 150, and $220 \mathrm{GHz}$ from 2009 onwards.

The SPT will finish a survey of $6 \%$ of the sky (2500 square degrees) in the winter of 2011 (middle panel of Figure 1). This region was selected for its extremely low level of galactic dust emission and visibility from the South Pole. The SPT dataset will yield a large catalog of SZdiscovered galaxy clusters, measurements of the small-scale Sunyaev-Zel'dovich (SZ) and CMB temperature power spectra, and a high-S/N detection of CMB lensing.

\section{Observations to Bandpowers}

The power spectrum analysis presented here uses data at $150 \mathrm{GHz}$ taken during the 2008 and 2009 austral winters. This includes five fields whose total area is 790 square degrees. Each field 

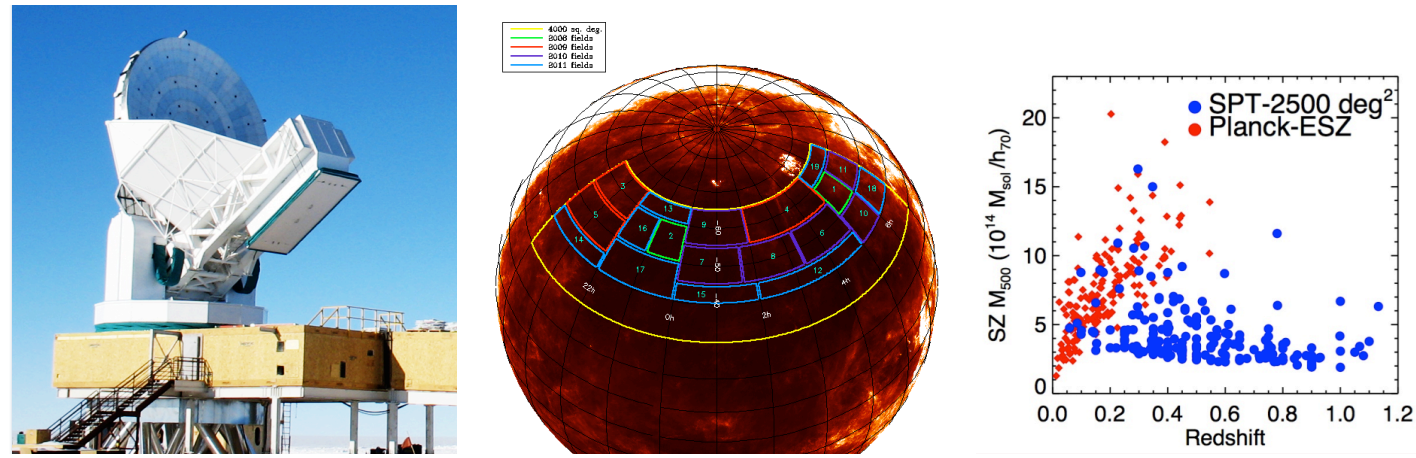

Figure 1: Left panel: The SPT is a 10-m telescope located at the South Pole and optimized for a large SZ galaxy cluster survey. Middle panel: The SPT survey overlaid on the IRAS dust map. The survey region was chosen for its exceptionally low galactic dust emission. The survey will finish observing $2500 \mathrm{deg}^{2}$ at 95,150 , and $220 \mathrm{GHz}$ by the end of 2011. The power spectrum discussed in this work is based on a third of the survey. Right panel: Galaxy cluster mass versus redshift for clusters in the Planck ESZ catalog or SPT catalog. Due to its smaller beam, the SPT survey is more sensitive to high redshift galaxy clusters.

was observed to an approximate depth of $18 \mu \mathrm{K}$-arcmin at $150 \mathrm{GHz}$. Details of the calibration, beam, and bandpower estimation can be found in $\mathrm{K} 11$; we outline the basics here.

We use a pseudo- $C_{\ell}$ method to estimate the bandpowers. In pseudo- $C_{\ell}$ methods, bandpowers are estimated directly from the spherical harmonic transform of the map after correcting for effects such as TOD filtering, beams, and finite sky coverage. We process the data using a cross spectrum based analysis to eliminate noise bias. Our scan strategy produces of order 100 complete but noisy maps of each field, and so is ideally suited to a cross-spectrum analysis. Before Fourier transforming, each map is apodized by a window designed to mask out $>50$ mJy point sources and to avoid sharp edges at the map borders. Beam and filtering effects are corrected for using simulations. We report the bandpowers in terms of $\mathscr{D}_{\ell}$, where

$$
\mathscr{D}_{\ell}=\frac{\ell(\ell+1)}{2 \pi} C_{\ell}
$$

The data is also subjected to a stringent set of jackknife tests to search for systematic errors. We find no significant evidence for systematic contamination of the SPT bandpowers.

The resulting bandpowers ${ }^{1}$ are shown in Fig. 2, along with other published data for comparison. The K11 bandpowers map out the damping tail of the CMB temperature power spectrum, and are the best measurement of the CMB power spectrum from the third to ninth acoustic peaks.

\section{Cosmological Implications}

We fit the SPT data using Monte Carlo Markov chain (MCMC) methods (see K11 for details). We include the seven-year WMAP (WMAP7, [1]) bandpowers in all MCMCs. Low redshift measurements of the Hubble constant $H_{0}$ using the Hubble Space Telescope [8] and the baryon acoustic oscillation (BAO) feature using SDSS and 2dFGRS data [9] are included in some results.

\footnotetext{
${ }^{1}$ http://lambda.gsfc.nasa.gov/product/spt
} 

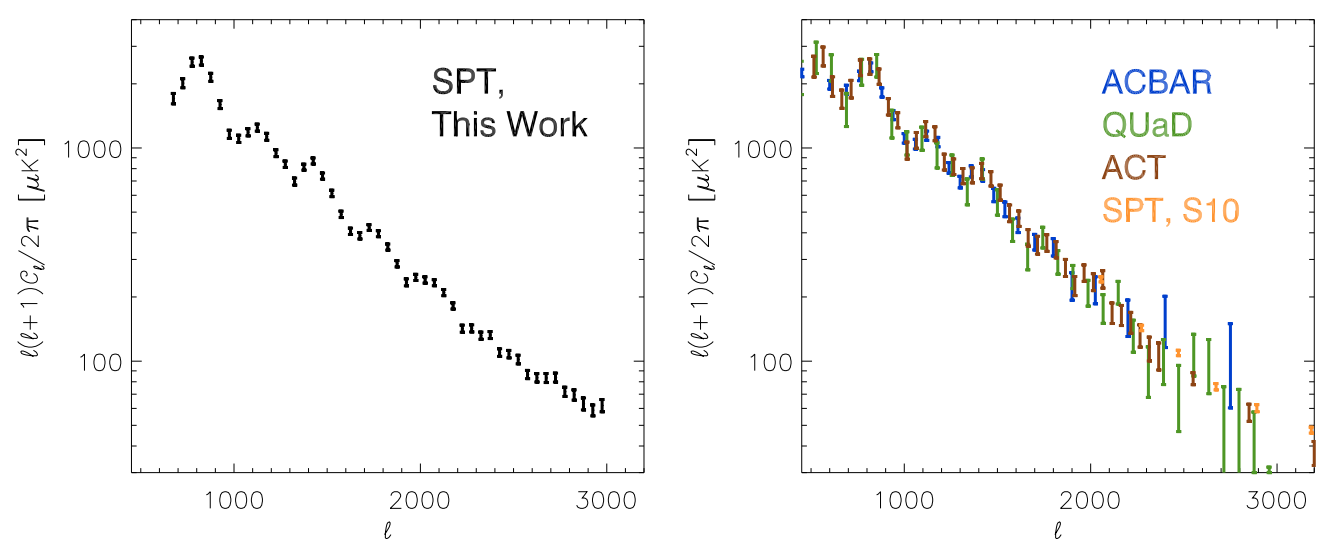

Figure 2: The SPT power spectrum from K11. The peak at $\ell \sim 800$ is the third acoustic peak. For comparison we show in the right panel other recent measurements of the CMB damping tail from ACBAR [3], QUaD [4], ACT [5], and SPT [7].
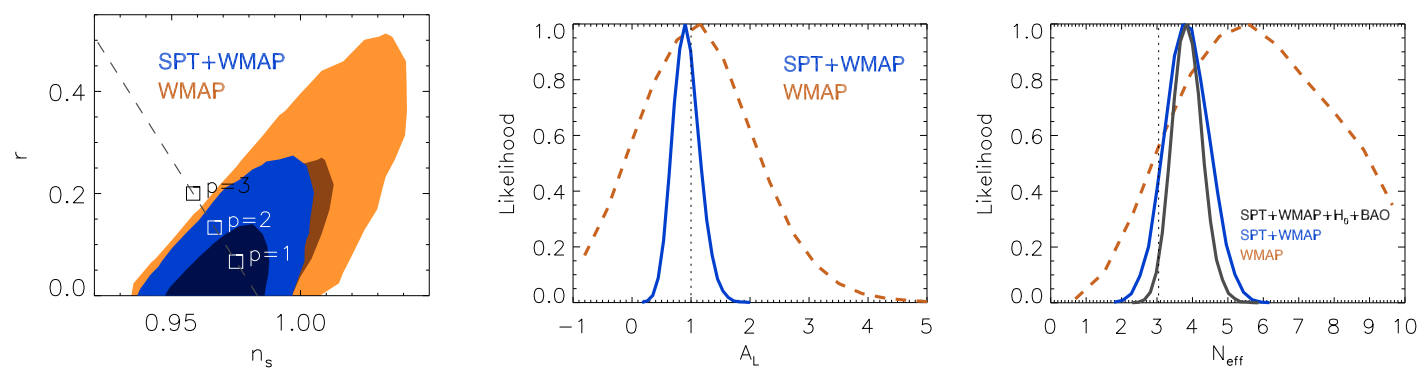

Figure 3: Left panel: Likelihood surfaces for $r$ and $n_{s}$ for the WMAP7 and WMAP7+SPT power spectra. The SPT data significant improve the limits on inflation. The line marks the prediction for chaotic inflationary models. Middle panel: Likelihood function for the magnitude of gravitational lensing potential. Right panel: Likelihood function for the number of relativistic species (e.g. neutrinos) with different data sets.

Our baseline cosmological model is a spatially flat, gravitationally lensed $\Lambda \mathrm{CDM}$ cosmology. In this model, the addition of the SPT data improves the constraints on $\Omega_{b} h^{2}$ and $n_{s}$ by $\sim 25 \%$, and the constraint on $\theta_{s}$ by nearly a factor of two.

The SPT small-scale power spectrum measurement significantly improves our ability to investigate extensions to the $\Lambda \mathrm{CDM}$ model. K11 explore a number of extensions, here we summarize the results on the tensor-to-scalar ratio, gravitational lensing, and neutrinos.

\subsection{Tensor-to-Scalar Ratio}

Inflation is expected to produce primordial tensor perturbations (i.e. gravitational waves). These perturbations imprint potentially detectable effects onto the CMB temperature and polarization spectra. The amplitude of the tensor spectrum is often given in terms of the tensor-to-scalar ratio, $r=\Delta_{h}^{2}\left(k_{0}\right) / \Delta_{R}^{2}\left(k_{0}\right)$ with pivot scale $k_{0}=0.002 \mathrm{Mpc}^{-1}$. A detection of $r$ would provide an extremely interesting window onto the energy scale of inflation.

In the long run, the best measurement of $r$ will come from B-mode CMB polarization. How- 
ever at the present, the strongest constraints come from the CMB temperature anisotropy. There is a degeneracy between $r$ and $n_{s}$ as shown in the left panel of Fig. 3. The CMB power at low multipoles increases as $r$ increases, but this effect can be partially cancelled by increasing $n_{s}$ and decreasing $\Delta_{R}^{2}$. The small-scale CMB measurements help to break this degeneracy. The SPT data together with WMAP7, BAO, and $H_{0}$ constrain $r<0.17$ (95\% CL), a factor of two improvement over WMAP7 alone. We also show the predictions for $r$ and $n_{s}$ from chaotic inflationary models with inflaton potential $V(\phi) \propto \phi^{p}$ and $N=60$, where $N$ is the number of e-folds between the epoch when modes that are measured by SPT and WMAP exited the horizon during inflation and the end of inflation. These models predict $r=4 p / N$ and $n_{s}=1-(p+2) / 2 N$. Models with $p \geq 3$ are disfavored at more than $95 \%$ confidence for $N \leq 60$.

\subsection{Gravitational Lensing}

The paths of $\mathrm{CMB}$ photons are distorted by the gravity of intervening matter as they travel from the surface of last scattering to us, a process referred to as gravitational lensing. Lensing alters the CMB temperature power spectrum at the few percent level. The acoustic peak structure is smoothed, and power is preferentially added to smaller angular scales. K11 parameterize the lensing amplitude by a factor $A_{L}$ which rescales the lensing potential power spectrum in a $\Lambda$ CDM cosmology. Since lensing encodes information on the distribution of matter at intermediate redshifts, the magnitude of lensing informs us about the neutrino mass, curvature, dark energy and modified gravity theories.

The SPT bandpowers accurately measure the acoustic peaks in the damping tail and are therefore sensitive to the lensing magnitude (middle panel of Fig. 3). The SPT + WMAP7 data measure $A_{L}^{0.65}=0.94 \pm 0.15$ (K11). The exponent of 0.65 is chosen to gaussianize the posterior likelihood. No lensing is rejected at $6 \sigma$. This is the best detection measurement of CMB lensing.

\subsection{Neutrinos}

In the standard theory of the early universe, there are three neutrino species that contribute $\sim 10 \%$ of the energy density at recombination. The effective number of particle species that are relativistic prior to and during recombination, $N_{\mathrm{eff}}$, ${ }^{2}$ is slightly higher (3.046) due to energy injection from electron-positron annihilation at the end of neutrino freeze-out. A significant detection of $N_{\text {eff }} \neq 3.046$ would point to the presence of extra relativistic species in the early universe.

The addition of extra relativistic species increases the expansion rate during the radiationdominated era. After fixing the quantities that are the robustly measured by WMAP, the main effect of this increased expansion rate is to increase the angular scale of photon diffusion and thereby lower power in the damping tail [10]. Hence measurements of the CMB damping tail in conjunction with WMAP constrain the number of relativistic species.

We extend the $\Lambda \mathrm{CDM}$ model by introducing $N_{\text {eff }}$ as a free parameter (right panel of Fig. 3). K11 find a preference $N_{\text {eff }}=3.046$ over no relativistic species at $7.5 \sigma$. [1] find $N_{\text {eff }}>2.7(95 \%$ CL) using WMAP7 alone, while the SPT+WMAP7 data constrain $N_{\text {eff }}$ to be $N_{\text {eff }}=3.85 \pm 0.62$ (K11). This constraint is $1.3 \sigma$ higher than the standard $N_{\text {eff }}=3.046$. When the $H_{0}$ and BAO data are added, the constraint tightens to $N_{\text {eff }}=3.86 \pm 0.42,1.9 \sigma$ higher than the standard value. For

\footnotetext{
${ }^{2} N_{\text {eff }}$ is defined such that $\rho_{v}=N_{\text {eff }} 7 / 8(4 / 11)^{4 / 3} \rho_{\gamma}$
} 
massless neutrinos, these high values of $N_{\text {eff }}$ are in tension with the observed number counts of lowredshift galaxy clusters - high $N_{\text {eff }}$ would underpredict the observed number counts. However, this tension vanishes when neutrinos are allowed to have mass. With massive neutrinos, the preferred $N_{\text {eff }}$ is similar at $3.97 \pm 0.43$.

\section{Conclusion}

We have reported on recent measurements of the damping tail of the CMB power spectrum using data from the SPT by K11. The SPT power spectrum uses $150 \mathrm{GHz}$ data and spans the multipole range $650<\ell<3000$, where it is dominated by primary CMB anisotropy. K11 combine this spectrum with data from WMAP7, $H_{0}$, and BAO to constrain extensions to the $\Lambda \mathrm{CDM}$ cosmological model. Here we have presented the salient results on the tensor-to-scalar ratio, gravitational lensing, and the number of neutrinos.

The SPT data presented here account for only a third of the full SPT-SZ survey. The full SPTSZ survey, which is expected to be completed by the end of 2011, will cover approximately 2500 square degrees. With $150 \mathrm{GHz}$ data of the quality used here and with additional data at 95 and 220 $\mathrm{GHz}$, a power spectrum analysis of the full SPT survey will be twice as sensitive as that presented here.

\section{References}

[1] E. Komatsu, K. M. Smith, J. Dunkley, C. L. Bennett, B. Gold, G. Hinshaw, N. Jarosik, D. Larson, M. R. Nolta, L. Page, D. N. Spergel, M. Halpern, R. S. Hill, A. Kogut, M. Limon, S. S. Meyer, N. Odegard, G. S. Tucker, J. L. Weiland, E. Wollack, and E. L. Wright, Seven-year Wilkinson Microwave Anisotropy Probe (WMAP) Observations: Cosmological Interpretation, ApJS 192 (Feb., 2011) 18-+, [arXiv:1001.4538].

[2] J. Silk, Cosmic black-body radiation and galaxy formation, ApJ 151 (1968) 459.

[3] C. L. Reichardt, P. A. R. Ade, J. J. Bock, J. R. Bond, J. A. Brevik, C. R. Contaldi, M. D. Daub, J. T. Dempsey, J. H. Goldstein, W. L. Holzapfel, C. L. Kuo, A. E. Lange, M. Lueker, M. Newcomb, J. B. Peterson, J. Ruhl, M. C. Runyan, and Z. Staniszewski, High-Resolution CMB Power Spectrum from the Complete ACBAR Data Set, ApJ 694 (Apr., 2009) 1200-1219, [arXiv:0801.1491].

[4] S. Gupta, P. Ade, J. Bock, M. Bowden, M. L. Brown, G. Cahill, P. G. Castro, S. Church, T. Culverhouse, R. B. Friedman, K. Ganga, W. K. Gear, J. Hinderks, J. Kovac, A. E. Lange, E. Leitch, S. J. Melhuish, Y. Memari, J. A. Murphy, A. Orlando, C. O’Sullivan, L. Piccirillo, C. Pryke, N. Rajguru, B. Rusholme, R. Schwarz, A. N. Taylor, K. L. Thompson, A. H. Turner, E. Y. S. Wu, M. Zemcov, and QUaD Collaboration, Parameter Estimation from Improved Measurements of the Cosmic Microwave Background from QUaD, ApJ 716 (June, 2010) 1040-1046, [arXiv:0909.1621].

[5] S. Das, B. D. Sherwin, P. Aguirre, J. W. Appel, J. R. Bond, C. S. Carvalho, M. J. Devlin, J. Dunkley, R. Dünner, T. Essinger-Hileman, J. W. Fowler, A. Hajian, M. Halpern, 
M. Hasselfield, A. D. Hincks, R. Hlozek, K. M. Huffenberger, J. P. Hughes, K. D. Irwin, J. Klein, A. Kosowsky, R. H. Lupton, T. A. Marriage, D. Marsden, F. Menanteau, K. Moodley, M. D. Niemack, M. R. Nolta, L. A. Page, L. Parker, E. D. Reese, B. L. Schmitt, N. Sehgal, J. Sievers, D. N. Spergel, S. T. Staggs, D. S. Swetz, E. R. Switzer, R. Thornton, K. Visnjic, and E. Wollack, Detection of the Power Spectrum of Cosmic Microwave Background Lensing by the Atacama Cosmology Telescope, Physical Review Letters 107 (July, 2011) 021301-+, [arXiv:1103.2124].

[6] R. Keisler, C. L. Reichardt, K. A. Aird, B. A. Benson, L. E. Bleem, J. E. Carlstrom, C. L. Chang, H. M. Cho, T. M. Crawford, A. T. Crites, T. de Haan, M. A. Dobbs, J. Dudley, E. M. George, N. W. Halverson, G. P. Holder, W. L. Holzapfel, S. Hoover, Z. Hou, J. D. Hrubes, M. Joy, L. Knox, A. T. Lee, E. M. Leitch, M. Lueker, D. Luong-Van, J. J. McMahon, J. Mehl, S. S. Meyer, M. Millea, J. J. Mohr, T. E. Montroy, T. Natoli, S. Padin, T. Plagge, C. Pryke, J. E. Ruhl, K. K. Schaffer, L. Shaw, E. Shirokoff, H. G. Spieler, Z. Staniszewski, A. A. Stark, K. Story, A. van Engelen, K. Vanderlinde, J. D. Vieira, R. Williamson, and O. Zahn, A Measurement of the Damping Tail of the Cosmic Microwave Background Power Spectrum with the South Pole Telescope, ApJ in press (May, 2011) [arXiv:1105.3182].

[7] E. Shirokoff, C. L. Reichardt, L. Shaw, M. Millea, P. A. R. Ade, K. A. Aird, B. A. Benson, L. E. Bleem, J. E. Carlstrom, C. L. Chang, H. M. Cho, T. M. Crawford, A. T. Crites, T. de Haan, M. A. Dobbs, J. Dudley, E. M. George, N. W. Halverson, G. P. Holder, W. L. Holzapfel, J. D. Hrubes, M. Joy, R. Keisler, L. Knox, A. T. Lee, E. M. Leitch, M. Lueker, D. Luong-Van, J. J. McMahon, J. Mehl, S. S. Meyer, J. J. Mohr, T. E. Montroy, S. Padin, T. Plagge, C. Pryke, J. E. Ruhl, K. K. Schaffer, H. G. Spieler, Z. Staniszewski, A. A. Stark, K. Story, K. Vanderlinde, J. D. Vieira, R. Williamson, and O. Zahn, Improved Constraints on Cosmic Microwave Background Secondary Anisotropies from the Complete 2008 South Pole Telescope Data, ApJ 736 (July, 2011) 61-+, [arXiv: 1012 .4788].

[8] A. G. Riess, L. Macri, S. Casertano, H. Lampeitl, H. C. Ferguson, A. V. Filippenko, S. W. Jha, W. Li, and R. Chornock, A 3\% Solution: Determination of the Hubble Constant with the Hubble Space Telescope and Wide Field Camera 3, ApJ 730 (Apr., 2011) 119-+, [arXiv:1103.2976].

[9] W. J. Percival, B. A. Reid, D. J. Eisenstein, N. A. Bahcall, T. Budavari, J. A. Frieman, M. Fukugita, J. E. Gunn, Ž. Ivezić, G. R. Knapp, R. G. Kron, J. Loveday, R. H. Lupton, T. A. McKay, A. Meiksin, R. C. Nichol, A. C. Pope, D. J. Schlegel, D. P. Schneider, D. N. Spergel, C. Stoughton, M. A. Strauss, A. S. Szalay, M. Tegmark, M. S. Vogeley, D. H. Weinberg, D. G. York, and I. Zehavi, Baryon acoustic oscillations in the Sloan Digital Sky Survey Data Release 7 galaxy sample, MNRAS 401 (Feb., 2010) 2148-2168, [arXiv: 0907.1660 ].

[10] Z. Hou, R. Keisler, L. Knox, M. Millea, and C. Reichardt, How Additional Massless Neutrinos Affect the Cosmic Microwave Background Damping Tail, ArXiv:1104.2333 (Apr., 2011) [arXiv:1104.2333]. 